\title{
PERFORMANCE EVALUATION OF INTEGRATED VOICE/DATA SERVICES OVER GSM/EGPRS WITH RADIO RESOURCE DIMENSIONING
}

\author{
Nawal EL-Fishawy, Hossam Ahmed and Noha Ramadan \\ Dept. of Commun., Faculty of Electronic Eng., Menouf, Egypt. \\ nelfishawy@hotmail.com, $\quad$ hossamkh@yahoo.com, \\ noha_ramadan@yahoo.com
}

(Received September 2, 2006 Accepted October 31, 2006)

\begin{abstract}
In this paper an analytical model for GSM/EGPRS is developed. It is based on the performance of $M / M / C / \infty / N$ queueing system with FCFS queue discipline. This model is adapted to guarantee GPRS/EGPRS system constraint and Internet traffic (ON/OFF traffic) over a single cell. The interaction of voice calls with GPRS/EGPRS connections is studied. Different performance parameters are calculated as blocking and delay probability, average throughput, average number of waiting users and queueing time. These performance parameters are obtained under different coding schemes $(C-S c)$ and three main radio resource strategies which are Complete Partitioning $(C P)$, Partial Sharing(PS), and Complete Sharing(CS). The obtained results show that the $(C P)$ strategy is more superior than the $(P S)$ and $(C S)$ strategies.
\end{abstract}

\section{INTRODUCTION}

General packet radio service(GPRS) complements Global System for Mobile Communications(GSM) to form together a $2.5 \mathrm{G}$ system. GPRS implements a packet switching network to the existing circuit switching network sharing the original Radio Link and Medium Access Control protocol(RLC/MAC) structure of GSM which now conveys voice and data services. Enhanced GPRS(EGPRS) is an evolution of GPRS, offering greater data rates in the same bandwidth by using nine different Modulation and Coding Schemes.

The challenge of the wireless network is to guarantee the desired quality of service $(\mathrm{Q} O S)$ requirements for both voice and data services. This can be achieved by radio resource dimensioning. For voice traffic over GSM, Erlang model [1] is still the main tool for resource dimensioning. GPRS/ EGPRS[2], [3] network is designed to transmit several data services such as WAP, Web, E-Mail, FTP, etc... The traffic corresponding to each of these services is characterized as ON/OFF process.

Most existing analytical models treating traffic over a communication system are classified to two classes. The first class corresponds to "open" vision of the cell. In this model, the traffic in the cell is generated by infinite number of users and can be modeled using queueing theory [4] or Markov Modulated Poisson Process (MMPP) [5] 
such that the arrival of traffic is Poissonian distribution. The second class corresponds to "closed" vision of the cell where the traffic is generated by finite number of users based on discrete time Markov chains[6], [7]or modified Engset model[8] which is a pure loss system model. In these models, the traffic is modeled by ON/OFF process following a geometric or general distribution.

In our study, a queueing model with finite users, channels, and infinite number of waiting positions over a closed cell is proposed. The data traffic is assumed to follow ON/OFF process with memoryless distribution, based on the actual GPRS/EGPRS system. Furthermore, the GSM/EGPRS performance parameters, e.g., delay , blocking probability, average queueing time and average throughput, in different radio resource allocation strategies are deduced. Also the effect of implementing different C-Sc are considered (see Table 1). Variable size of the web page and the reading time are considered.

Table 1. GPRS Coding Schemes [8].

\begin{tabular}{|l|c|c|c|c|}
\hline GPRS Coding schemes & C-Sc-1 & C-Sc-2 & C-Sc-3 & C-Sc-4 \\
\hline RLC block radio(bytes) & 23 & 33 & 39 & 53 \\
\hline Data rate: $\boldsymbol{\mu}_{\text {GPRS }}(\mathbf{k b} / \mathbf{s})$ & 9.05 & 13.4 & 15.6 & 21.4 \\
\hline
\end{tabular}

The paper is organized as following: in section 2, the main features of GPRS/EGPRS system are described; section 3 shows a brief explanation of the characteristics of ON/OFF data traffic model; section 4 describes the proposed analytical model and the derivation of the performance parameters; in section 5 the numerical results are discussed; and finally the paper is concluded in section 6 .

\section{THE MAIN FEATURES OF GPRS/EGPRS SYSTEM}

The GPRS/EGPRS architecture is very similar to GSM. The main difference is the implementation of packet switching for data transmission instead of circuit switching, which introduced some new components including Serving GPRS Support Node(SGSN), Gateway GPRS Support Node(GGSN) and Base Station Controller (BSC) which must be equipped with GPRS hardware Packet Control Unite(PCU) and software to support the (RLC/MAC).

Time slots used by GPRS/EGPRS are called the packet data channel (PDCH). The basic transmission unit of a PDCH is called a radio block. Nine different coding schemes have been defined for EGPRS. Each radio block is coded using one of these coding schemes. They may be used alternatively, depending on the quality of the radio interface.

To support the packet switching principle of GPRS/EGPRS, the resources of one $\mathrm{PDCH}$ are assigned temporarily to one mobile station (MS). The BSC transmits in each downlink radio block header an Uplink State Flag(USF) notifying the MS with the downlink Temporary Block Flow(TBF). TBF is a physical connection used to support the transfer of a number of blocks. Each TBF is addressed by a Temporary Flow Identity(TFI) assigned by the network. When a TBF is assigned, the MS is informed of 
which time slot(s) to use and its TFI address (5 bit length) so that multiplexing of blocks originated from different MSs on the same PDCH is possible. However, there is a system limit on the maximum number of MSs that can have a simultaneously downlink TBF( 32 per TDMA).

\section{CHARACTERISTICS OF VOIC/ DATA TRAFFIC MODEL}

Data sources such as WWW browsing, FTP and e-mail are elastic traffic i.e. the packets can be transmitted at any rate up to the limit of system capacity. These services are handled by GPRS/EGPRS. In the case of WWW source, the traffic generated by a session is characterized by ON/OFF process where the ON periods corresponds to the flow of E-mail messages or web pages load. The size of the web page, $\alpha$ (bits), is the size of the file which can be downloaded during the ON period. After downloading a WWW document (ON period), the user is consuming certain amount of time for reading the information (OFF period). This time interval is called reading time, $\tau(\mathrm{sec})$, (see Figure 1).

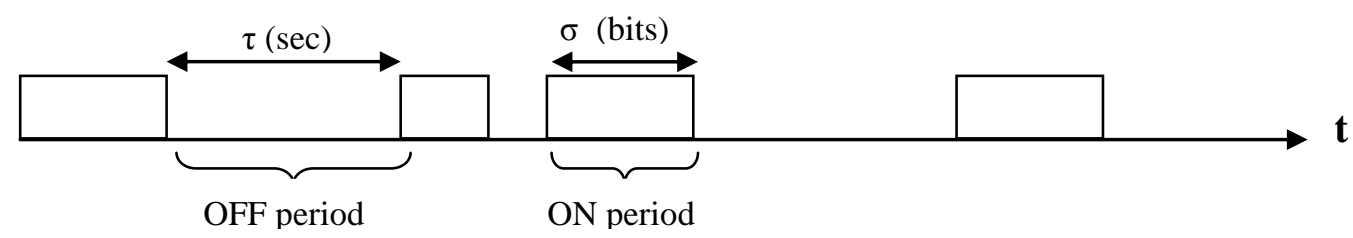

Figure 1: Typical characteristic of www service.

The Internet traffic (both ON and OFF periods) have shown that heavy-tailed distributions characterize these traffics such as Pareto, Weibull and LogNormal [9], [10]. Many researches such as [11] compares Pareto and memoryless distributions for both ON sizes and OFF times and get that the average performance parameters are largely insensitive to the exact input distribution, only the means of the file size and think time duration are used in the formulas and the memoryless distributions are the most convenient choice. So we can use the M/M/C/oo/N FCFS queue in terms of exponential distribution (memoryless) and apply the solution to any other distribution with the same mean.

\section{SYSTEM MODEL}

Our model is based on $\mathrm{M} / \mathrm{M} / \mathrm{C} / \infty / \mathrm{N}$ FCFS discipline. The model is adapted to GPRS/EGPRS system constraints which take into consideration the maximum number of users that can simultaneously have a downlink TBF and the maximum number of users that can use a single time slot. We focus our study on resources employed for downlink because of the nature of data traffic. All data users generate the same type of traffic i.e. Web or WAP. Each user generates an infinite number of ON/OFF sessions with exponential distribution for the ON sizes and OFF times (insensitive property). We also considered a finite number of users in a single cell and there are no retransmissions at the MAC/ RLC layer i.e. ideal SNR and BER. 
Three main strategies allocating the radio resources are considered:

I. Complete Partitioning (CP)

II. Partial Sharing (PS)

III. Complete Sharing (CS)

\section{I- CP Strategy}

With CP strategy, the total channels are divided into two fixed parts between voice and data traffic i.e. T is the total time slots, $\mathrm{C}_{\mathrm{d}}$ is the number of PDCHs for GPRS, $\mathrm{C}_{\mathrm{v}}$ is the number of TCHs for GSM such that $\mathrm{C}_{\mathrm{v}}=\mathrm{T}-\mathrm{C}_{\mathrm{d}}$. N is the total number of data users in the cell, $\mathrm{m}$ is the maximum number of GPRS users that can use a single time slot and $\mathrm{n}_{\max }$ is the maximum number of GPRS users in active transfer and is obtained by [8]

$$
n_{\max }\left(C_{d}\right)=\min \left(N, 32, m C_{d}\right)
$$

There are $\mathrm{N}$ users, each one alternates between $\mathrm{ON}$ and OFF periods. ON period size has exponential distribution with mean $\mathrm{E}[\sigma]$ and OFF period duration has exponential distribution with mean $E[\tau]$. Among $\mathrm{N}$ users a random number $\mathrm{j}(\mathrm{t})$ are in the $\mathrm{ON}$ state at time $\mathrm{t}$ where $j(t)=0,1,2, \ldots, N$. This stochastic process describes the number of users in progress so $j(t)$ is a birth and death process with birth and death rates of $\lambda_{\mathrm{j}}$ and $\mu_{\mathrm{j}}$ (see Figure 2) respectively, which are given by:

$$
\begin{aligned}
& \lambda_{j}=(N-j) \alpha \\
& \mu_{j}=\left\{\begin{array}{ll}
j \mu_{G P R S} & 0 \leq j \leq n_{\max }-1 \\
n_{\max } \mu_{G P R S} & n_{\max } \leq j \leq N
\end{array}\right\}
\end{aligned}
$$

where $\alpha=E[\sigma] / E[\tau]$

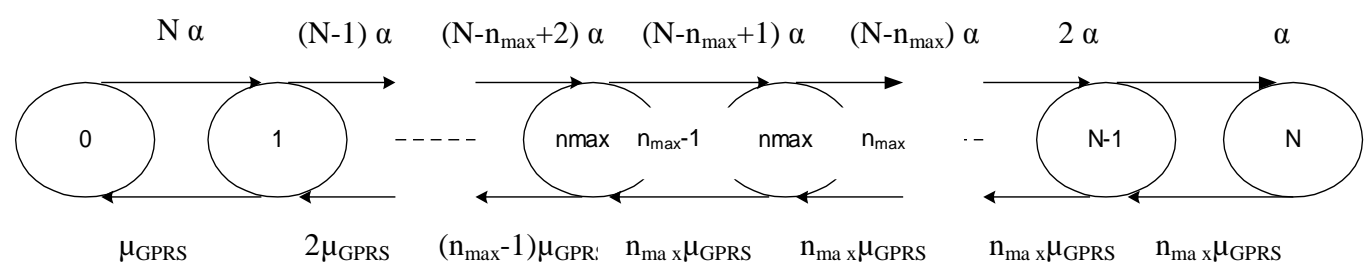

Figure 2: State transition diagram of GPRS users.

Let $P(j)$ be the steady state probability that $\mathrm{j}$ users are in active transfer, which is obtained by solving the steady state equilibrium equation of the birth and death process :

$$
P(j)=\left\{\begin{array}{cc}
P_{0} \rho^{j} C_{N}^{j} & 0 \leq j \leq n_{\max }-1 \\
P_{0} \rho^{j} C_{N}^{j} \cdot \frac{j !}{n_{\max } !} \cdot n_{\max }^{n_{\max }-j} & n_{\max } \leq j \leq N
\end{array}\right\}
$$


where $\rho=(E[\sigma] / E[\tau]) \mu_{G P R S}^{-1}$

and $P_{0}$ is obtained by the normalization condition as :

$$
P_{0}^{-1}=\sum_{i=0}^{n_{\max }-1} \rho^{i} C_{N}^{i}+\sum_{i=n_{\max }}^{N} \rho^{i} C_{N}^{i} \frac{i !}{n_{\max } !} n_{\max }^{n_{\max }-i}
$$

Based on (4), other performance parameters can be derived. A new arrival is accepted into the system only if the number of GPRS users in the system(in queue plus those in service) below the maximum accepted number $\mathrm{N}$ otherwise the new arrival is blocked, thus the blocking probability is

$$
P_{B}\left(C_{d}\right)=P_{0} \rho^{N} \cdot \frac{N !}{n_{\max } !} \cdot n_{\max }^{n_{\max }-N}
$$

The arriving GPRS user is accepted to transmit/receive data if a sufficient number of free recourses are available otherwise it is delayed, thus the delay probability is

$$
P_{D}=\sum_{j=n_{\max }}^{N} P(j)=P_{0} \sum_{j=n_{\max }}^{N} \rho^{j} C_{N}^{j} \cdot \frac{j !}{n_{\max } !} \cdot n_{\max }^{n_{\max }-j}
$$

The average queue length is identical to the average number of users in the system

$$
L_{q}\left(C_{d}\right)=N_{s}\left(C_{d}\right)=\sum_{j=n_{\max }+1}^{N}\left(j-n_{\max }\right) P(j)=\sum_{j=0}^{N} j P(j)
$$

The average number of new arrivals is

$$
\begin{aligned}
\bar{\lambda}=\sum_{j=0}^{N} \lambda_{j} P(j) & \\
= & \mathrm{E}[\sigma] / \mathrm{E}[\tau]\left[N \sum_{j=0}^{N} P(j)-\sum_{j=0}^{N} j P(j)\right] \\
& =\left[N-L_{q}\left(C_{d}\right)\right] \mathrm{E}[\sigma] / \mathrm{E}[\tau]
\end{aligned}
$$

In our case the average throughput obtained by each user in the system is the amount of data that can be transmitted successfully in a given amount of time and can be obtained as [4]

$$
T H\left(C_{d}\right)=\bar{\lambda}\left(1-P_{B}\left(C_{d}\right)\right) / L_{q}\left(C_{d}\right)
$$

The average number of waiting users is equal to the average number of users in the system excluding the average number of users in active transfer and is given by

$$
N_{w}\left(C_{d}\right)=N_{s}\left(C_{d}\right)-\bar{\lambda} / \mu_{G P R S}
$$

Using Little's Law with (9) and (11), the average waiting time can be get as: 


$$
T_{q}\left(C_{d}\right)=\frac{N_{w}\left(C_{d}\right)}{\bar{\lambda}}=\frac{L_{q}\left(C_{d}\right)}{E[\sigma] / E[\tau]\left[N-L_{q}\left(C_{d}\right)\right]}-1 / \mu_{G P R S}
$$

\section{II- PS Strategy}

Data users have their exclusive bandwidth but they can also use the available bandwidth of voice service with pre-emptive service priority for voice calls. Among the $T$ available time slots a number $\mathrm{C}_{\mathrm{d}}$ is permanently reserved as PDCHs for GPRS (static PDCHs) whereas the remaining $\left(\mathrm{C}_{v}=\mathrm{T}-\mathrm{C}_{\mathrm{d}}\right)$ time slots are shared between the two services with pre emption for the voice service (dynamic PDCHs) where:

$$
n_{\text {max }}(C)=\min (N, 32, m C)
$$

where $\mathrm{C}$ is the static and dynamic PDCHs. Since the service time of voice calls is much greater than the service time of GPRS users, so the decomposition technique [4], [12] can be used. The essential point for this technique is the use of the voice call steady-state probability to describe the interaction of voice calls with GPRS connections.

We have assumed that voice calls arrive as a Poisson process with arrival rate $\lambda_{\mathrm{v}}$ and their call holding time is a negative exponential distribution with service rate $\mu_{\mathrm{v}}$. We further assume that, the network only dedicates one time slot per voice user and if all the channels are in use by other voice users the call will be blocked. Using Erlang B formula, the steady-state probability distribution for voice calls is:

$$
R(n)=\frac{\rho_{v}^{n} / n !}{\sum_{i=0}^{T-C_{d}} \rho_{v}^{i} / i !} \quad \text { for } \mathrm{n}=0,1, \ldots, \mathrm{T}-\mathrm{C}_{\mathrm{d}}
$$

where $_{v}=\lambda_{v} / \mu_{v}$

The channels unused by the voice services may be used for the data services. The probability that $\zeta$ time slots are available for data transfer equal to the probability that $\left(\mathrm{C}_{\mathrm{v}}-\zeta\right)$ time slots are used by GSM voice calls. Put $\mathrm{x}=\left(\mathrm{C}_{\mathrm{v}}-\zeta\right)$ in (14) we get:

$$
R(\xi)=\frac{\rho_{v}^{x} / x !}{\sum_{i=0}^{C v} \rho_{v}^{i} / i !}
$$

Following the same procedure considered in evaluating the performance parameters of $\mathrm{CP}$, we could get the average queue length as:

$$
L_{q}(C)=\sum_{\xi=0}^{C v} R(\xi) L_{q}\left(C_{d}+\xi\right)
$$

The average blocking probability as: 


$$
P_{B}(C)=\sum_{\xi=0}^{C v} R(\xi) P_{B}\left(C_{d}+\xi\right)
$$

The average throughput as:

$$
T H(C)=\bar{\lambda}\left(1-P_{B}(\xi)\right) / L_{q}(C)
$$

The average number of waiting users (queue length) can be get as

$$
N_{w}(C)=\sum_{\xi=0}^{C v} R(\xi) N_{w}\left(C_{d}+\xi\right)
$$

The average waiting time as:

$$
T_{q}(C)=\sum_{\xi=0}^{C v} R(\xi) T_{q}\left(C_{d}+\xi\right)
$$

\section{III- CS strategy}

In CP and PS strategies, GPRS users use a fixed number of time slots, which are not shared with GSM voice users. Therefore, the QoS of GSM users that are circuit switched is decreased. Here, we propose a complete sharing policy that prevents decrease in QoS for GSM users. In this policy, the data packets are permitted using all available free channels $(\eta)$ that are not already in use by voice users where

$$
n_{\max }(\eta)=\min (N, 32, m \eta)
$$

Among (T) time slots which are not used by the voice calls there are $\eta$ time slots may be used for data traffic. The probability that $\eta$ time slots are available for data transfer equal to the probability that $(T-\eta)$ time slots are used by GSM voice calls. Put $y=(T-\eta)$ and substitute in (14) we get the probability of $(\mathrm{T}-\eta)$ time slots used by GSM voice calls as:

$$
R(\eta)=\frac{\rho_{v}^{y} / y !}{\sum_{i=0}^{T} \rho_{v}^{i} / i !} \quad \text { for } \eta=0,1, \ldots, \mathrm{T}
$$

The performance parameters of the CS strategy can calculated by the following equations:

The average queue length is

$$
L_{q}(T)=\sum_{\eta=0}^{T} R(\eta) L_{q}(\eta)
$$

The average blocking probability of GPRS users is:

$$
P_{B}(T)=\sum_{\xi=0}^{C_{v}} R(\eta) P_{B}(\eta)
$$


The average throughput is:

$$
T H(T)=\bar{\lambda}\left(1-P_{B}(T)\right) / L_{q}(T)
$$

The average number of waiting users(queue length) can be get as:

$$
N_{w}(T)=\sum_{\eta=0}^{T} R(\eta) N_{w}(\eta)
$$

The average waiting time is:

$$
T_{q}(T)=\sum_{\eta=0}^{T} R(\eta) T_{q}(\eta)
$$

\section{NUMERICAL RESULTS AND DISCUSSIONS}

\subsection{The Effect Of The Web Page Size (ON Period)}

We will study the effect of the web page size on the queue length and the delay time of $\mathrm{CP}$ strategy. Figure 3 shows the effect of increasing the size of the web page from $10 \mathrm{kB}$ to $40 \mathrm{kB}$ on the average queue length when the number of data users changes from 1 to 70, four PDCH available for GPRS users and the reading time is $12 \mathrm{sec}$. Our results show that the web page size has a great effect on the performance parameters, where increasing the web page size, increases the service time of each active user. So, the number of waiting users increases as the size of the web page increases as shown in the figure. Also the average delay time increases with the increase of the web page size (see Figure 4).

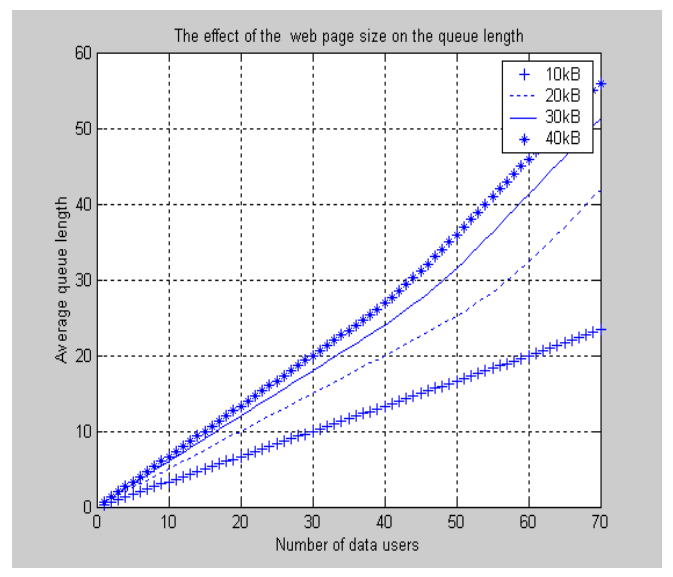

Figure 3. The effect of the web page size on the queue length for CP strategy.

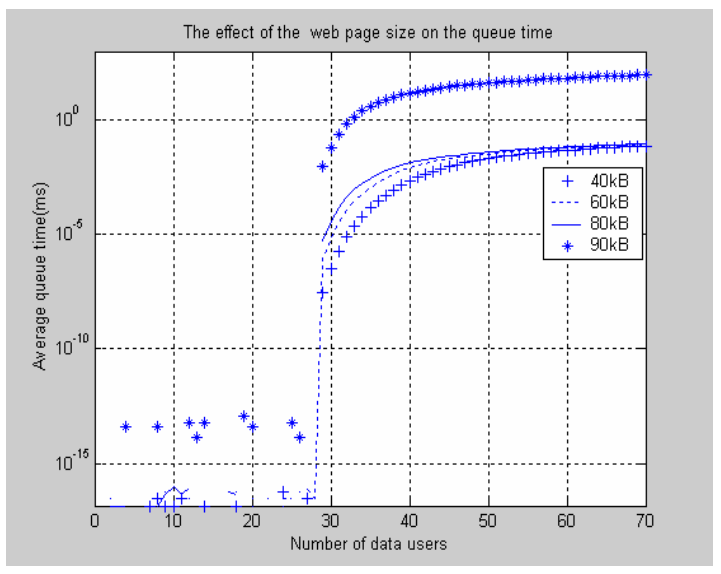

Figure 4. The effect of the web page size on the queue time for CP strategy. 


\subsection{The Effect Of The Reading Time (OFF Period)}

Figures 5 and 6 show the effect of the reading time on the queue length and the queueing time respectively. When the user spends more time for studying the information i.e., reading a web page, no data are transferred during this OFF period, (i.e. no downloaded traffic), and hence the delay time and the number of waiting users are decreased as increasing the reading time.

\subsection{The Coding Schemes Effect}

We will examine the performance parameters for complete partitioning strategies under four different coding schemes. The average web page size considered are $30 \mathrm{kB}, 10 \mathrm{kB}$, and $5 \mathrm{kB}$ and the average reading time is $12 \mathrm{~s}$. The number of time slots available for GPRS users are 4 time slots. We assume that up to 7 users can share a single time slot $(\mathrm{m}=7)$.

Figure 7 shows the average delay versus the number of data users as deduced in Eq.(19). We notice that as the coding scheme increases the average delay decreases. This is because of the higher data rate of the C-Sc-4 than the other coding schemes (see Table1). The average delay increases as the number of data users increases but no delay observed before $\mathrm{N}$ equal 32, 5 bit length of TFI address, where the number of data users is below the maximum available number in the $\operatorname{system}\left(\mathrm{n}_{\max }\right)$. Figures $\mathbf{8}$ and 9 highlight the average queue length and the total average throughput for different coding schemes respectively (Eqs. (16), (18)). As the coding scheme increases the transfer rate of the download data is increased and then every user is getting service faster. This led to the rapid service of the waiting users and so the decrease of the queue length. The average throughput for each user in our case is rather constant and equal to the data rate $\left(\mu_{\mathrm{GPRS}}\right)$, while the throughput value increases as the coding scheme increases.

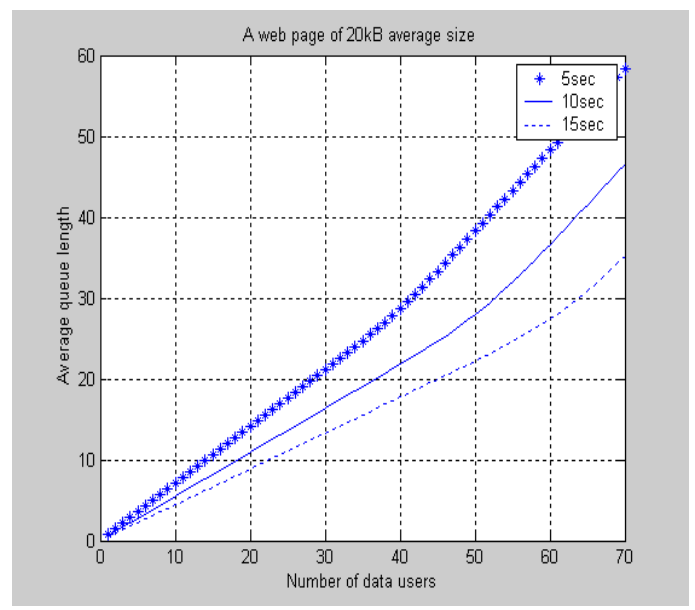

Figure 5. The effect of the reading time on the queue length for CP strategy.

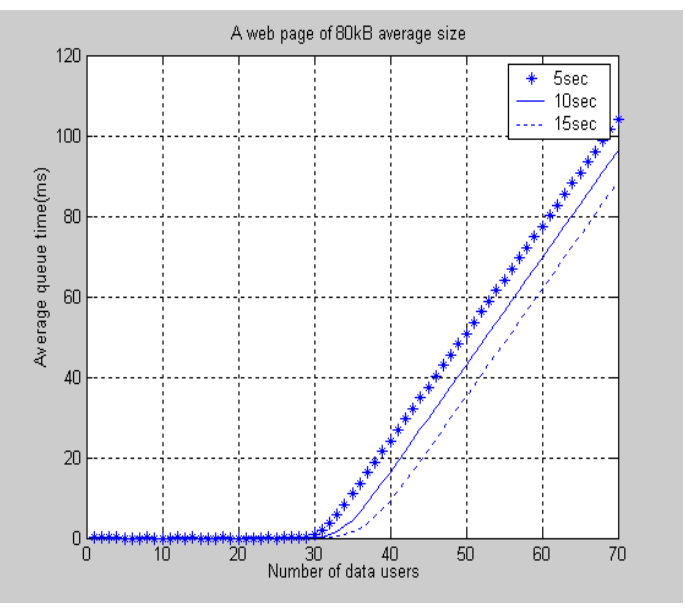

Figure 6. The effect of the reading time on the queue time for CP strategy. 


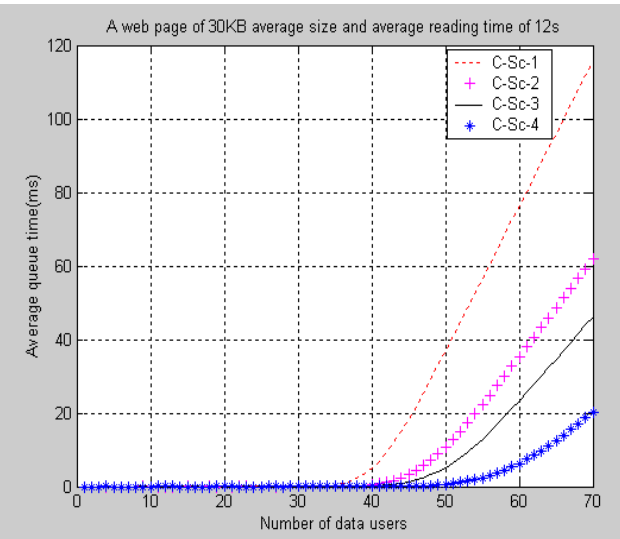

Figure 7. The effect of the coding schemes on the queue time for $\mathrm{CP}$ strategy.

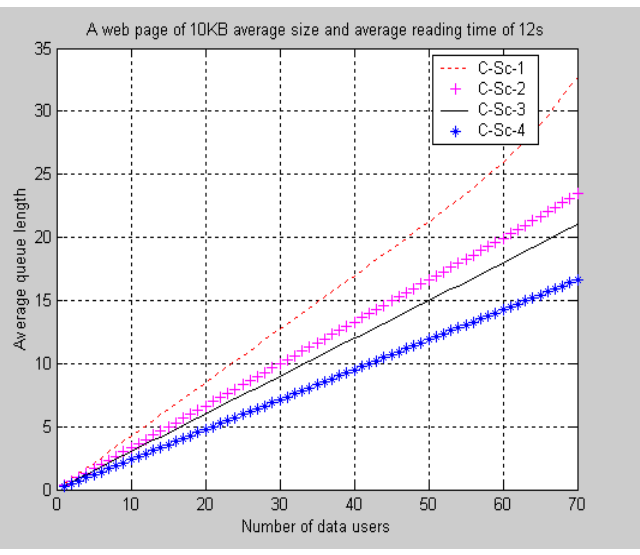

Figure 8. The effect of the reading time on the queue length for CP strategy.

\subsection{Comparison Between The Three Different Strategies}

In this part we focus on comparing the performance parameters of the three radio resource strategies, where the queueing time, the queue length and the throughput are shown in Figures 10, 11 and $\mathbf{1 2}$ respectively, for C-Sc-2.. The figures are obtained with a number of GPRS users equals 30 , the average web page size is $5 \mathrm{kB}$, the blocking probability for voice traffic is $2 \%$, the number of static PDCHs is 2 and the total number of channels equal $14\left(\mathrm{C}_{\mathrm{v}}=12\right)$. By comparing the results of the three different strategies, it is clear that the complete partitioning has the best results in terms of the least delay and queue length and the highest throughput while the partial and complete sharing have the worst performance.

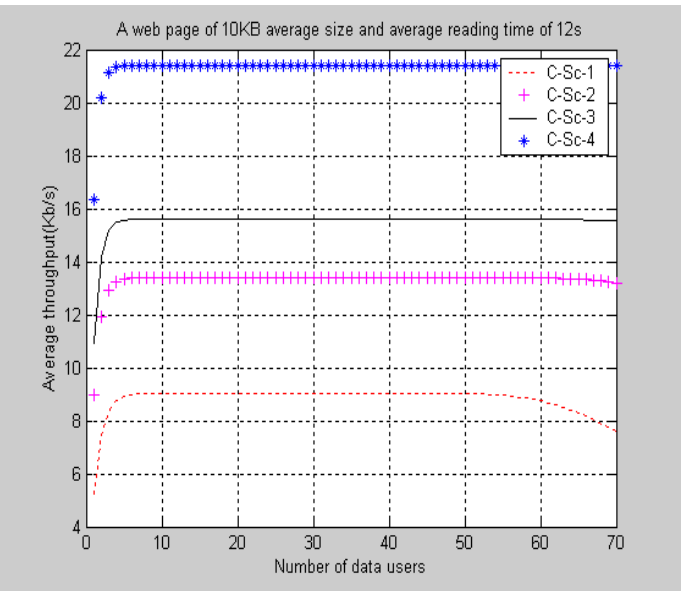

Figure 9. The effect of the coding schemes on the throughput for CP strategy.

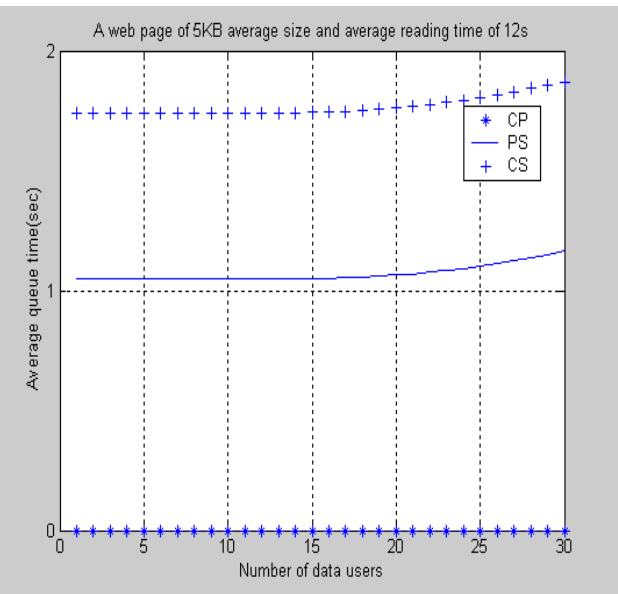

Figure 10. Comparing the queue time for CP, PS, CS strategies. 


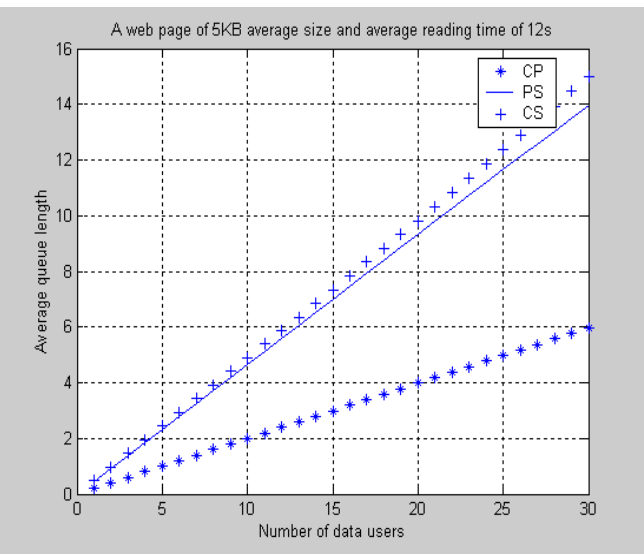

Figure 11. Comparing the queue length for CP,PS and CS strategies

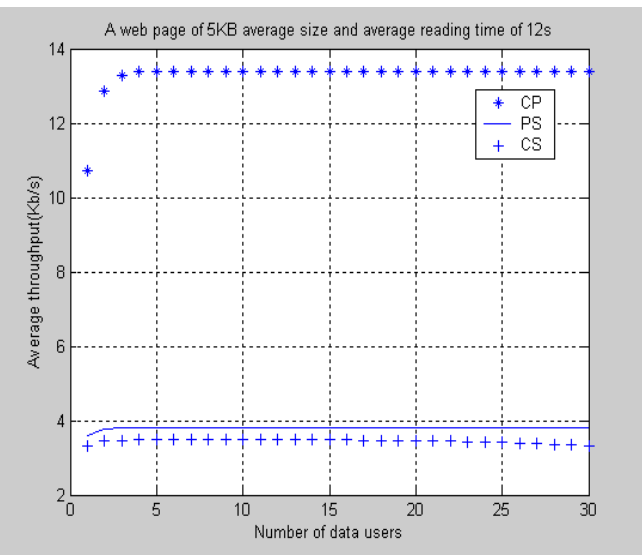

Figure 12. Comparing the throughput for $\mathrm{CP}, \mathrm{PS}$ and CS strategies

\section{CONCLUSION}

In this paper, an analytical model for measuring the performance parameters for integrated voice/data services over the air interface for GSM/EGPRS system using the delay system for data users and loss system for voice users is proposed. Our study based on a simple mathematical model over a closed cell and a limited number of users. Three radio resource allocation strategies are examined and compared. The results show that the $(\mathrm{CP})$ strategy has the best performance such that the queueing delay, the queue length, and the average throughput are more encouraging for $\mathrm{CP}$ than that of PS and CS strategies. The proposed model is more practical and close to the real system in comparison with the models which consider an infinite number of users and open vision of the cell.

\section{REFERENCES}

[1] Leonard Kleinrock, "Queueing Systems", Vol. 1,J. Wiley \& Sons, 1975.

[2] ETSI TS 101344 , "Digital cellular telecommunications system (Phase 2+) ; General Packet Radio Service (GPRS) Service description; Stage 2(3GPP TS 03.60 version 7.9.0 Release 1998)" V7.9.0 (2002-09).

[3] file:///Y/Vanliga/Ericsson/www/read/info.htm

[4] S. Ni and S. Haggman, "GPRS Performance Estimation in GSM Circuit Switched services and GPRS Shared Resource Systems", WCNC, Vol. 3, pp. 1417-1421, 1999.

[5] Xiaoyan Fang and Dipak Ghosal, "Analyzing Packet Delay Across a GSM/GPRS Network", Examination Report under Section 18 (3) issued in GB 00251363.3 application by the United Kingdom Patent Office on Jul. 24, 2003, pp. 1-10.

[6] B. Baynat and P. Eisenmann, "Towards an Erlang-like Law for GPRS/EDGE Network Engineering". In proc. of IEEE ICC, Paris, France, June 2004. 
[7] K. Boussetta, B. Baynat and P. Eisenmann, "Performance evaluation of GPRS/EDGE networks: A novel discrete-time Markov model", 5th World Wireless Congress (WWC 2004) San Francisco, USA - May, 2004.

[8] H. Dahmouni, B. Morin and S. Vaton and, "Performance Modelling of GSM/GPRS Cells with Different Radio Resource Allocation Strategies", IEEE WCNC, New Orleans, 2005.

[9] R. Kalden and S. Ibrahim, "Searching for Self-Similarity in GPRS". The 5th annual Passive \& Active Measurement Workshop, PAM 2004, France, April 2004.

[10] Fischer, M. J., D. M. B. Masi, P. H. Brill, D. Gross, and J. Shortle, "Using the Correct Heavy-Tailed Arrival Distribution in Modeling Congestion Systems", The 11th International Conference on Telecommunication Systems Management, Naval Postgraduate School, Monterey, CA, October 2-5, 2003.

[11] S. Ben Fredj, T. Bonald, A. Prouti `ere, G. R'egni' e, J.W. Roberts" Statistical bandwidth sharing: a study of congestion at flow level", ACM Sigcomm, 2001.

[12] F. Delcoigne, A. Proutière and G. Régnié," Modeling integration of streaming and data traffic", Performance Evaluation, 55 (3-4), pp 185- 209, 2004.

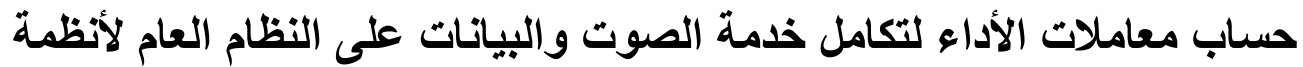 المحمول (GSM) ونظام خدمات الر اديو للحزم العامة المتقدمة (EGPRS)

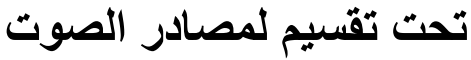

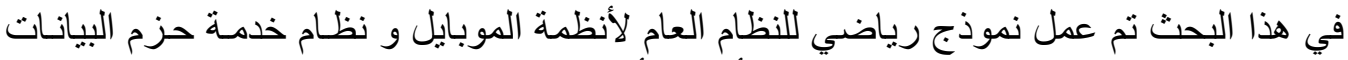

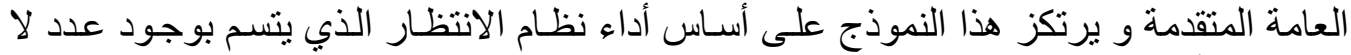

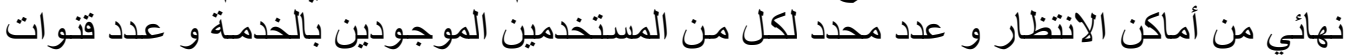

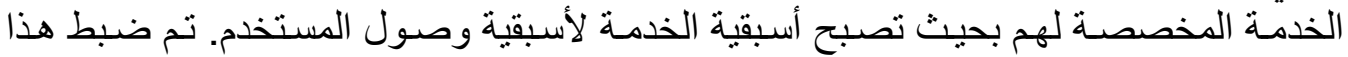

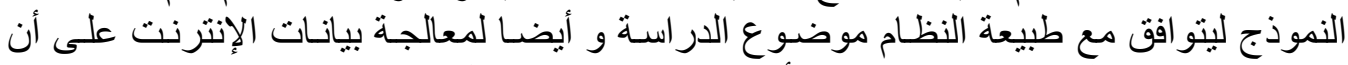

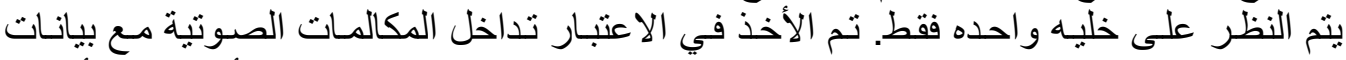

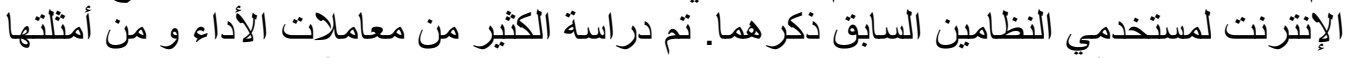

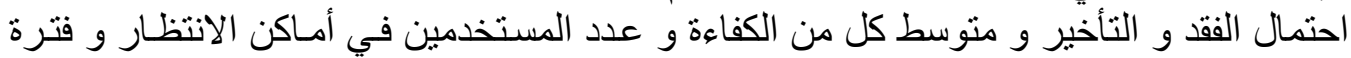

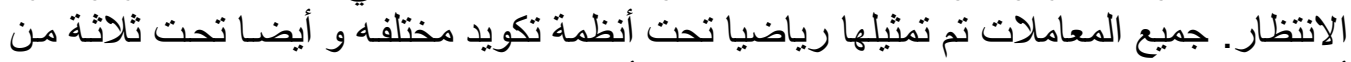

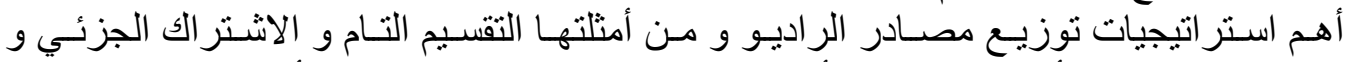

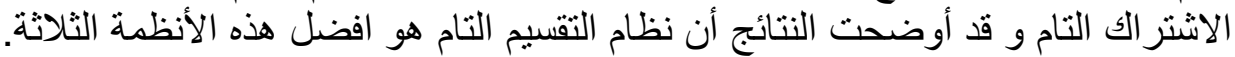

